\title{
Mesenchymal Stem Cells-derived Exosomes as Probable Triggers of Radiation-induced Heart Disease
}

\section{Lan Luo}

Xuzhou Medical University https://orcid.org/0000-0001-7549-9852

\section{Chen Yan}

Nagasaki University: Nagasaki Daigaku

\section{Naoki Fuchi}

Nagasaki University

\section{Yukinobu Kodama}

Nagasaki University Hospital: Nagasaki Daigaku Byoin

\section{Xu Zhang}

Nagasaki University: Nagasaki Daigaku

\section{Goto Shinji}

Nagasaki University: Nagasaki Daigaku

\section{Miura Kiyonori}

Nagasaki University Hospital: Nagasaki Daigaku Byoin

\section{Hitoshi Sasaki}

Nagasaki University Hospital: Nagasaki Daigaku Byoin

Tao-Sheng Li ( $\sim$ litaoshe@nagasaki-u.ac.jp )

Nagasaki University - Bunkyo Campus: Nagasaki Daigaku

\section{Research Article}

Keywords: Mesenchymal stem cells, Exosomes, lonizing radiation, Heart disease

Posted Date: March 1st, 2021

DOI: https://doi.org/10.21203/rs.3.rs-263552/v1

License: (1) (i) This work is licensed under a Creative Commons Attribution 4.0 International License. Read Full License

Version of Record: A version of this preprint was published at Stem Cell Research \& Therapy on July 22nd, 2021. See the published version at https://doi.org/10.1186/s13287-021-02504-5. 


\section{Abstract}

Background: Radiation-induced heart disease have been reported, but the mechanisms remain unclear. Mesenchymal stem cells (MSCs), also resident in heart are highly susceptible to radiation. We examined the hypothesis that altered secretion of exosomes from MSCs as the triggers of radiation-induced heart disease.

Methods: By exposing human placental tissue-derived MSCs to 5 Gy $\mathrm{Y}$-rays, we will then isolate exosomes from the culture medium $48 \mathrm{~h}$ later and use to evaluate the quantity and quality changes of exosomes from MSCs after radiation exposures. The biological effects of exosomes from irradiated MSCs on HUVEC and H9c2 cells were also examined.

Results: Although the amount and size distribution of exosomes did not differ between the non-irradiated and irradiated MSCs, miRNA sequences indicated many up- or down-regulated miRNAs in irradiated MSCs-exosomes. In vitro experiments using HUVEC and H9c2 cells showed that irradiated MSCsexosomes significantly decreased cell proliferation, but increased cell apoptosis and DNA damage. Moreover, irradiated MSCs-exosomes impaired the tube formation of HUVEC cells and induced calcium overload of $\mathrm{H} 9 \mathrm{c} 2$ cells.

Conclusions: Exosomes released from irradiated MSCs shows an altered miRNA profiling and harmful effect to damage heart cells, which provides new insight on the mechanism of radiation-related heart disease risks.

\section{Background}

In the 1960s, clinicians first recognized that cardiovascular complications occurred in patients who underwent radiotherapy (RT) for chest tumors (1). The following studies involving patients who received relatively high thoracic RT doses demonstrated an excess risk of radiation-induced heart disease (RIHD) (2-4). The incidence of RIHD considerably declined with decreased cardiac radiation exposures using modern RT techniques (intensity-modulated RT, image-guided RT, etc.) (5). Indeed, Darby and van den Bogaard et al. identified that the mean heart doses linearly correlated with the risk of $\operatorname{RIHD}(5,6)$. However, the minimum threshold dose remained unclear. Epidemiological data revealed increased heart disease risks in atomic bomb survivors with individually estimated dose over $0.5 \mathrm{~Gy}$ (7). Besides, individuals with other traditional risk factors (e.g., hypertension, hyperlipidemia, diabetes mellitus, smoking), or at a young age, seemed to be more vulnerable to develop RIHD (8). The rising radiation exposure potentiality by medical imaging, galactic cosmic, or terrestrial ionizing resources makes it reasonable to worry about RIHD.

The clinical presentations of RIHD are currently well documented, including coronary artery atherosclerosis, pericarditis, cardiomyopathy, and conduction defects (9). RIHD mostly takes years or decades to manifest, making it challenging to interpret the underlying cellular and molecular mechanisms. Now, we know that ionizing radiation (IR) causes endothelial dysfunction and inflammatory 
responses, preceding the development of atherosclerosis, cardiac fibrosis, and tissue remodeling (9). And sustained DNA lesions, oxidative stress, mitochondria dysfunction, epigenetic regulations, and telomere erosions are also related to the development of RIHD (10-12). Notably, these molecular changes interact with each other and act diversely in different types of heart cells. Therefore, the pathophysiological mechanisms on RIHD are still asked to precisely understand.

Classical radiobiology identifies that cells with high proliferative rates and immature features are more susceptible to IR. Cardiomyocytes, the primary cell type in heart, are postmitotic that incapable of proliferating. Hence, the dogma that heart as a radioresistant organ has last for a long time. However, apart from the cardiomyocytes, other cells like microvascular endothelial cells, fibroblasts, and lately identified mesenchymal stem cells (MSCs) (13) also reside in heart. According to classical radiobiology (14), MSCs theoretically reveal higher radiosensitivity than other matured heart tissue cells like endothelial cells and cardiomyocytes. In response to different stimuli, MSCs release abundant exosomes, which are essential mediators of intercellular communication (15). Hypoxia-primed bone marrow MSCs promoted cardiac function in a mouse model of myocardial infarction via upregulated exosomal miR$125 \mathrm{~b}$-mediated cell protection (16). Thus, we hypothesize that radiation exposure alters the secretion of exosomes from MSCs, which subsequently induces the damage of other heart tissue cells with less radiosensitivity.

By exposing human placenta-derived mesenchymal stem cells (hp-MSCs) to 5 Gy $\mathrm{Y}$-rays, we herein investigated the radiation-induced change on the secretion of exosomes from hp-MSCs. We further in vitro evaluated the potential role of exosomes from irradiated hp-MSCs in regulating the survival and function of heart tissue cells.

\section{Methods}

\section{Culture of hp-MSCs, HUVEC, and H9c2 cells}

hp-MSCs were obtained from as a gift (17). hp-MSCs were maintained in Dulbecco's modified Eagle's medium (DMEM) (Wako, Osaka, Japan) supplemented with 10\% fetal bovine serum (FBS, Hyclone Laboratories, Logan, UT, USA), $10 \mathrm{ng} / \mathrm{ml}$ human recombinant basic fibroblast growth factor (Wako), and $1 \%$ penicillin $(100 \mathrm{U} / \mathrm{ml}) /$ streptomycin $(100 \mathrm{U} / \mathrm{ml})$ solution (Life Technologies). The HUVEC cell line was purchased from PromoCell GmbH (Germany) and grown in endothelial cell growth medium 2 (PromoCell) supplemented with $10 \%$ fetal bovine serum and $1 \%$ penicillin /streptomycin. The H9c2 cell line was purchased from ATCC (CRL-1446) and grown in DMEM supplemented with $10 \%$ fetal bovine serum and $1 \%$ penicillin /streptomycin. All Cells were cultured in a $5 \% \mathrm{CO}_{2}$ incubator at $37^{\circ} \mathrm{C}$.

\section{Radiation exposure}

The culture medium from twice-passaged hp-MSCs culture at $80 \%$ confluence was aspirated, cells were washed with phosphate buffered saline (PBS) (Wako, Osaka, Japan) to remove the residual FBS. Fresh culture medium supplemented with 10\% exosome-depleted FBS (System Biosciences) was added. Then 
the hp-MSCs were exposed to 0 or 5 Gy $\mathrm{Y}$-rays at a dose rate of $1 \mathrm{~Gy} / \mathrm{min}$ using a PS-3100SB $\mathrm{Y}$-ray irradiation system with a Cs source (Pony Industry Co., Ltd. Osaka, Japan) (18).

\section{Isolation of exosomes derived from non-irradiated and irradiated hp-MSCs}

After 48 hours of incubation, culture medium from hp-MSCs that irradiated or not was collected and underwent different steps of centrifugation as previously described with minor modifications (19). Briefly, culture medium was centrifuged at $300 \mathrm{~g}$ for 3 minutes, at $4{ }^{\circ} \mathrm{C}$ and $2000 \mathrm{~g}$ for 30 minutes to remove cell debris and apoptotic bodies. The supernatant was ultra-centrifuged at $4{ }^{\circ} \mathrm{C}$ and $100000 \mathrm{~g}$ for 120 minutes to collect exosomes. Then, the pellet was washed with PBS and underwent another step of ultracentrifugation at $4{ }^{\circ} \mathrm{C}$ and $100000 \mathrm{~g}$ for 120 minutes to concentrate and purify exosomes. At last, the pellet was re-suspended in PBS and went through $0.22 \mu \mathrm{m}$ filter (Millex) for further experiments or stored at $-80^{\circ} \mathrm{C}$.

\section{Exosomes characterization}

Image of exosomes were taken by a transmission electron microscope. Briefly, $5 \mathrm{ul}$ exosomes were dropped on the copper net and incubated at room temperature (RT) for 5 minutes. Then, excess liquids were removed by the filter paper. Add $5 \mathrm{ul} 1 \%$ phosphotungstic acid to the copper mesh and incubated for 1 minute at RT. Excess liquids were also removed by the filter paper. Add deionized water to the copper mesh to remove excess dye solution. Observe the exosomes under microscope after drying at RT.

The protein concentration of exosomes was tested as described in the instructions (Thermo Scientific 23235). Expression of exosome marker CD63 and TSG101 (System Biosciences) were verified western blot analysis. Exosome miRNAs were analyzed using gene chip miRNA 4.0 by the Filgen company.

\section{Uptake of exosomes}

Exosomes derived from non-irradiated (Non-irradiated-exo) and irradiated hp-MSCs (Irradiated-exo) were labeled with the PKH26 Red Fluorescent Cell Linker Kit (Sigma-Aldrich) according to the manufacturer's protocol with minor modifications. Non-irradiated or irradiated-exo diluted in PBS were added to $1 \mathrm{ml}$ Diluent C (Sigma-Aldrich). In parallel, $4 \mu \mathrm{PKH} 26$ dye was added to $1 \mathrm{ml}$ Diluent $\mathrm{C}$ and incubated with the exosome solution for 4 minutes. To bind excess dye, $2 \mathrm{ml} 0.5 \%$ BSA/PBS was added. The labeled exosomes were washed at 100,000 g for 1 hour, and the exosome pellet was diluted in $100 \mu \mathrm{l}$ PBS and used for uptake experiments. PKH26 labeled non-irradiated or irradiated-exo were cultured with HUVEC and $\mathrm{H} 9 \mathrm{c} 2$ cell line, respectively. The images of exosomes uptake were taken when co-culture at 3 and 24 hours using confocal microscopy.

\section{Evaluation of cell proliferation and DNA damage}

To evaluate the effects of exosomes on cell proliferation and DNA damage, HUVEC and H9c2 cells were seeded on 4-well chamber culture slides. After 72 hours of culture with non-irradiated-exo or irradiatedexo, the cells were washed with PBS and fixed in 4\% paraformaldehyde for 10 minutes. After blocking, the 
cells were incubated with KI67 Monoclonal Antibody (SolA15, Invitrogen), anti-53BP1 antibody (ab36823, Abcam), or anti-gamma H2A.X (ab2893, Abcam), followed by associated Alexa flour 488-conjugated second antibody. Nuclei were stained with DAPI, and the positively stained cells were counted under fluorescence microscopy with 200 -fold magnification, and 20 fields per section were randomly selected for quantitative counting.

\section{Annexin-V flow cytometry}

To evaluate the effects of exosomes on HUVEC and H9c2 cell apoptosis, HUVEC and H9c2 cells were seeded on $10 \mathrm{~cm}$ culture dishes. After 48 hours of culture with non-irradiated-exo or irradiated-exo, the cells were collected and washed with cold D-PBS by centrifugation for $5 \mathrm{~min}$ at $500 \times \mathrm{g}$ at $4{ }^{\circ} \mathrm{C}$. Cells treated with $3 \%$ formaldehyde in buffer for $30 \mathrm{~min}$ were included as positive control. The cell pellets were suspended with 100ul cold D-PBS, then added 5 ul of Annexin V-FTIC solution and $2.5 \mathrm{ul}$ dissolved PI as described in the manual (Beckman coulter). The samples were kept on ice and incubated for $10 \mathrm{~min}$ in the dark. Finally, 400ul ice-cold $1 \times$ binding buffer were added to the samples for analyze by flow cytometry.

\section{Tube formation}

Corning ${ }^{\circledR}$ Matrigel $\circledast$ Matrix (356230) was thawed overnight on ice at $4{ }^{\circ} \mathrm{C}$ according to the guidelines in the manual. All pipets and procedures were previously kept on ice. Added 289 ul chilled corning Matrigel matrix in to 24 -well culture plates avoiding air bubbles. Plates were incubated at $37^{\circ} \mathrm{C}$ for $30-60$ minutes. The medium remained was removed carefully without disturbing the matrix layer and the plates were ready to use. HUVEC cells were previously co-cultured with non-irradiated-exo or irradiated-exo for 48 hours. $300 \mathrm{ul}$ cell suspensions were collected and added to each well and incubated at $37^{\circ} \mathrm{C}, 5 \% \mathrm{CO}_{2}$ atmosphere. The tube formation was observed and pictured. The photo was further analyzed by Image J.

\section{Intracellular Calcium detection}

The intracellular calcium was examined by loading H9c2 cells with Fluo 3 (Dojindo Molecular Technologies, Inc) according to the instructions. Briefly, H9c2 cells were previously co-cultured with nonirradiated-exo or irradiated-exo for 48 hours. Cells were harvested ant then plated on 96-well plates. Culture medium were carefully removed without injuring the cells. Cells were washed with PBS gently and then incubated with loading buffer at $37^{\circ} \mathrm{C}$ for 1 hour. Loading buffer was removed carefully and added warm recording medium. The fluorescence was examined by multifunctional microplate detector.

\section{Statistical analysis}

All the results are presented as the mean \pm SD. The statistical significance was determined by one-way ANOVA and followed by Turkey's multiple comparisons test (GraphPad Prism). Differences were considered significant when $P<0.05$. 


\section{Results}

\section{Characterization of hp-MSCs and hp-MSCs exosomes}

Primarily expanded hp-MSCs exhibited a fibroblast-like morphology (Fig. 1A), and were identified as the biological properties of MSCs according to their expression pattern on cell surface markers of CD44, CD105, CD90, CD73, CD45, and CD34 (Fig.1 B, C). To investigate the impact of IR on the exosomal secretion, hp-MSCs were exposed to $5 \mathrm{~Gy}$ y-rays and the medium were collected $48 \mathrm{~h}$ later for exosomes isolation by ultracentrifugation. The successful isolation of exosomes was confirmed by the electron microscope image (Fig.1 D), nanoparticle track analysis (Fig.1 E), and western blot analysis on the expression of membrane markers of CD63 and TSG101 (Fig.1 F). The size distribution (Fig.1 E) and protein concentration (Fig.1 G) were not obviously different between the exosomes from the nonirradiated and irradiated MSCs. These data indicated very limited changes on the amount and size distribution of exosomes from hp-MSCs within $48 \mathrm{~h}$ after the exposure to $5 \mathrm{~Gy} \mathrm{Y}$-rays.

\section{Exosomes from non-irradiated and irradiated hp-MSCs exhibited different miRNAs expression profile}

We further measured the expression of miRNAs in exosomes by gene chip miRNA 4.0. In contrast to the less changes on the secretion amount and size distribution, miRNA sequences indicated many miRNAs in irradiated MSCs-exosomes were up- or down-regulated when compared to that of the non-irradiated hpMSCs (Fig.1 H). The top 20 miRNAs of up- (Table 1.) or down-regulation (Table 2.) in exosomes from irradiated hp-MSCs were listed up in table. Only miR-4655-5p were upregulated over 3-fold, but miR-4443, miR-7110-5p, miR-520g-3p, miR-382-5p, miR-424-3p, miR-3197, miR-6824-5p were downregulated over 3fold in irradiated hp-MSCs exosomes.

\section{Uptake of exosomes by HUVEC and H9c2 cells}

Then, we evaluated the biological effects of exosomes from the non-irradiated and irradiated hp-MSCs on endothelial cells and cardiomyocytes. By culturing HUVEC and H9c2 cells with the supplement of PKH26labeled exosomes in medium, the uptakes of exosomes by cells were observed using a confocal microscope. Red fluorescence was clearly detectable in the cytoplasm at $3 \mathrm{~h}$, and further enhanced after

$24 \mathrm{~h}$ (Fig.2 A, B). However, the uptakes of exosomes from either non-irradiated or irradiated hp-MSCs were observed quite similar by HUVEC and H9c2 cells. This demonstrated that the exosomes from the nonirradiated or irradiated hp-MSCs could be internalized by the HUVEC and H9c2 cells.

\section{Exosomes from irradiated hp-MSCs significantly impaired the survival of HUVEC and H9c2 cells}

The impact of non-irradiated and irradiated hp-MSCs exosomes on cell proliferation and DNA damage was observed after $72 \mathrm{~h}$ of co-culture using immune-fluorescence assay (Fig.3 A, D). The KI67 expression in HUVEC and H9c2 cells was increased by the non-irradiated hp-MSCs exosomes but decreased by the irradiated hp-MSCs exosomes (Fig.3 B, C). Due to the different specificities of antibodies between of HUVEC and H9c2 cells, DNA damage of cells was detected by the formation of 53BP1 or $\mathrm{Y}-\mathrm{H} 2 \mathrm{AX}$ foci in nuclei, respectively. Exosomes from non-irradiated hp-MSCs significantly decreased the percentages of 
cells with 53BP1 or $\mathrm{Y}-\mathrm{H} 2 \mathrm{AX}$ foci than that of control (Fig.3 E, F). However, the percentages of cells with 53BP1 or $\mathrm{Y}-\mathrm{H} 2 \mathrm{AX}$ foci were significantly increased by exosomes from irradiated hp-MSCs than that of non-irradiated hp-MSCs (Fig.3 E, F). All these results indicated that irradiated hp-MSCs exosomes impaired the proliferation and induced DNA damage in HUVEC and H9c2 cells.

Cell apoptosis was also evaluated after $48 \mathrm{~h}$ of co-culture using Annexin-V flow cytometry assay (Fig.4 A, D). The non-irradiated hp-MSCs exosomes significantly protected the HUVEC and H9c2 cells from apoptosis (Fig.4 B, E). However, the apoptosis of HUVEC and H9c2 cells was less decreased by exosomes from the irradiated hp-MSCs than those from non-irradiated hp-MSCs (Fig.4 B, E). Also, the necrosis of HUVEC cells was less decreased by exosomes from the irradiated hp-MSCs than those from nonirradiated hp-MSCs (Fig.4 C). However, both the non-irradiated and irradiated hp-MSCs exosomes did not affect the necrosis of H9c2 cells (Fig.4 F). In contrast to the non-irradiated hp-MSCs exosomes, irradiated hp-MSCs exosomes showed very poor ability to protect HUVEC and H9c2 cells from apoptosis.

\section{Exosomes from irradiated hp-MSCs revealed functional impairments to HUVEC and H9c2 cells}

To evaluate the potential roles of exosomes from irradiated hp-MSCs on cell function, we observed tube formation of HUVEC cells (Fig.5 A) and calcium transient of H9c2 cells (Fig.5 D) $48 \mathrm{~h}$ after co-culture. The tube formation of HUVEC cells was significantly increased by exosomes from the non-irradiated hp-MSCs but slightly decreased by exosomes from the irradiated hp-MSCs (Fig.5 B, C). However, the calcium transient of H9c2 cells was significantly increased by exosomes from the irradiated hp-MSCs, but did not change significantly by exosomes from the non-irradiated hp-MSCs (Fig.5 D). These results indicated the functional impairments of irradiated hp-MSCs exosomes to HUVEC and H9c2 cells.

\section{Discussions}

Emerging findings have identified the contribution of stem cell injury in radiation-induced tissue toxicity $(20,21)$. Exosomes seem to be essential mediators of communication between MSCs and heart cells (22). Resident MSCs in heart are known to play an essential role in cardiac homeostasis (23), and its dysfunction may contribute to heart disease development (24). Thus, following radiation exposure, the relatively high radiosensitive MSCs may secrete specific exosomes to induce injury of heart tissue cells, including cardiomyocytes and endothelial cells, which finally develop heart disease. To verify our hypothesis, we exposed hp-MSCs to $5 \mathrm{~Gy}$ Y-rays and then evaluated how the exosomes from irradiated hp-MSCs affect the biological properties of HUVEC and H9c2 cells.

We successfully isolated exosomes from the non-irradiated or irradiated hp-MSCs culture medium using ultracentrifugation, confirmed by electron microscopy and expressions of CD63 and TSG101. Although the similar amount and size distribution, exosomes from the non-irradiated and irradiated hp-MSCs revealed large different in the expressions of miRNAs, indicating radiation-induced alternation of exosomes secreting MSCs in quality rather than in quantity. 
MSCs-derived exosomes have been demonstrated to possess comparative regenerative potential as MSCs (25). We next investigated the potential effects of exosomes on heart tissue cells using HUVEC and H9c2 cells. Correspondingly, exosomes from non-irradiated hp-MSCs clearly showed the beneficial effects on cell proliferation, DNA damage, and cell apoptosis. In contrast, exosomes from irradiated hp-MSCs revealed much less beneficial, and sometimes even harmful effects to HUVEC and H9c2 cells. We were also engaged in identifying whether the exosomes from irradiated hp-MSCs would impair the functions of HUVEC and H9c2 cells. MSCs-derived exosomes promote angiogenesis via transferring signals to endothelial cells (26). The tube formation of HUVEC cells was facilitated by exosomes from nonirradiated hp-MSCs, but not the irradiated hp-MSCs. The homeostasis of calcium transient is a crucial factor for maintaining normal cardiac rhythm (27). Following ischemia/reperfusion injury, the internal levels of calcium in $\mathrm{H} 9 \mathrm{c} 2$ cells increased in a time-dependent manner (28), and calcium overload in $\mathrm{H} 9 \mathrm{c} 2$ cells may further accelerate reperfusion injury (29). We found that calcium transient in $\mathrm{H} 9 \mathrm{c} 2$ cells was significantly enhanced by exosomes from only the irradiated hp-MSCs, indicating the induction of calcium overload in cells. All these data suggested the harmful, rather than beneficial effects of exosomes from irradiated MSCs to heart cells.

Using miRNA sequence, we could find extensive changes on miRNAs between exosomes from nonirradiated and irradiated hp-MSCs. Among the upregulated miRNAs in the exosomes from irradiated hpMSCs, roles of miR-129-5p, miR-212-5p, miR-3120-5p, miR-16-1-3p, miR-4638-5p, miR-2392, and miR-324$3 p$ have been reported mostly about cancer development, but rarely about cardiovascular diseases. Geng et al. found that high fat diet-induced upregulation of miR-129-5p contributes to atherosclerosis development via beclin-1 inhibition (30). Zhao et al. identified that IgE activates miR-212-5p in asthma mice and causes declined blood tension via downregulating vascular NCX1 expression (31). Li et al. explored that miR-3120-5p interacted with LncRNA WTAPP1 suppressing endothelial progenitor cell migration and angiogenesis by decreasing MMP-1 levels and inhibiting PI3K/Akt/mTOR pathway (32). Ge et al. reported that miR-324-3p promoted high glucose-induced renal fibrosis via activation of MAPK and ERK1/2 pathways (33). Among the downregulated miRNAs in the exosomes from the irradiated hpMSCs, roles of miR-4443, let-7a-5p, miR-382-5p, miR-424-3p, miR-3197, miR-3178, miR-32-3p, miR-1273g$3 p$, miR-23a-5p, miR-29a-3p, miR-146a-5p, miR-517a-3p, and miR-516b-5p have been reported also mostly in cancer progression. Besides, the roles of miR-1273g-3p, miR-23a-5p, miR-29a-3p, and miR-146a-5p in heart disease development are broadly studied. Guo et al. figured that miR-1273g-3p promotes HUVEC cell dysfunction caused by acute glucose fluctuation (34). It showed that miR-23a-5p enhances atherosclerotic plaque progression (35) and hepatic fibrosis $(36,37)$. Yet, Lu et al. identified that miR-23a$5 p$ enriched in bone marrow-derived M2 macrophages with a reparative potential (38). miR-29a-3p is known to reduce cardiac hypertrophy $(39,40)$ and ischemia reperfusion injury $(41)$. miR-146a-5p is reported to attenuate ischemia/reperfusion injury via downregulating Irak1 and Traf6 and consequent blunted toll-like receptor signaling (42). miR-146a-5p deficiency in doxorubicin treated mice develop more severe cardiotoxicity (43). Importantly, exosomes from cardiosphere-derived cells have an abundant expression of miR-146a-5p, conferring cardiac regenerative therapeutic effects (44). However, Oh et al. miR-146a-5p were enriched in extracellular vesicles isolated from failing hearts reducing cardiac 
contractility via suppressing SUM01/SERCA2a signaling (45). In contrast, Fang et al. found that patients with upregulated serum levels of miR-29a-3p and miR-146a-5p are more likely to develop diffuse myocardial fibrosis (46). Thus, the exact roles of miR-23a-5p, miR-29a-3p, and miR-146a-5p need more indepth investigation.

This study has several limitations (but not limited to) that need further addressing. First, as a proof-ofconcept of study, we used MSCs from human placental tissues instead of the resident MSCs of heart because of the availability of cell sources. It is not very clear about the similarity of MSCs from different tissues/organs. Second, hp-MSCs were exposed only $5 \mathrm{~Gy}$ at high dose rate (1 Gy/min) in this study. As the biological effects to cells will be largely varied depending on the dose and dose rate of radiation, (47, 48), it is needed to evaluate the quantity and quality of exosomes from MSCs by exposing the cells to different dose and dose rate of radiation. Third, we only analyzed the miRNAs, but radiation exposure may also change other components such as lipids, proteins, and IncRNAs in exosomes. Otherwise, we did not try to further confirm the role of each up- or down-regulated miRNA in exosomes from irradiated MSCs to HUVEC and H9c2 cells.

\section{Conclusions}

In all, although the number of exosomes secreted from hp-MSCs was not changed by 5 Gy $\mathrm{Y}$-rays exposure, exosomes from the irradiated hp-MSCs caused damages to HUVEC and H9c2 cells. Our preliminary data from in vitro study has demonstrated that exosomes from MSCs may indirectly contribute to radiation-induced heart disease. Further studies, including interventional experiments and in vivo mice models are required to confirm our concept.

\section{Declarations}

\section{Ethics approval and consent to participate}

Not applicable.

\section{Consent for publication}

Not applicable.

\section{Availability of data and materials}

All the datasets used and/or analyzed during this study are available from the corresponding authors on reasonable request.

\section{Competing interests}

The authors declare that they have no competing interests. 


\section{Funding}

This work was supported by a Grant-in-Aid from the Ministry of Education, Science, Sports, Culture and Technology, Japan (17F17425), the National Natural Science Foundation of China (Grant No. 81802086), the Natural Science Foundation of Jiangsu Province (Grant No. BK20180995), the Specialized Research Fund for Senior Personnel Program (Grant No. D2019028) and the Young Science and Technology Innovation Team (Grant No. TD202005) of Xuzhou Medical University. The funders had no role in study design, data collection, and analysis, decision to publish, or preparation of the manuscript.

\section{Authors' contributions}

L.L. and TS.L. conceived and designed the experiments. L.L., C.Y., N.F., Y.K., X.Z., S.G., M. K., H.S., and TS.L. performed the experiments and analyzed the data. L.L. and TS.L. wrote the main manuscript text.

\section{Acknowledgements}

Not applicable.

\section{References}

1. COHN KE, STEWART JR, FAJARDO LF, HANCOCK EW. Heart disease following radiation. Medicine. 1967;46(3):281-98.

2. Dess RT, Sun Y, Matuszak MM, Sun G, Soni PD, Bazzi L, et al. Cardiac Events After Radiation Therapy: Combined Analysis of Prospective Multicenter Trials for Locally Advanced Non-Small-Cell Lung Cancer. J Clin Oncol. 2017;35(13):1395-402.

3. Hooning MJ, Botma A, Aleman BM, Baaijens MH, Bartelink H, Klijn JG, et al. Long-term risk of cardiovascular disease in 10-year survivors of breast cancer. J Natl Cancer Inst. 2007;99(5):365-75.

4. Castellino SM, Geiger AM, Mertens AC, Leisenring WM, Tooze JA, Goodman P, et al. Morbidity and mortality in long-term survivors of Hodgkin lymphoma: a report from the Childhood Cancer Survivor Study. Blood. 2011;117(6):1806-16.

5. van den Bogaard VA, Ta BD, van der Schaaf A, Bouma AB, Middag AM, Bantema-Joppe EJ, et al. Validation and Modification of a Prediction Model for Acute Cardiac Events in Patients With Breast Cancer Treated With Radiotherapy Based on Three-Dimensional Dose Distributions to Cardiac Substructures. J Clin Oncol. 2017;35(11):1171-8.

6. Darby SC, Ewertz M, McGale P, Bennet AM, Blom-Goldman U, Bronnum D, et al. Risk of ischemic heart disease in women after radiotherapy for breast cancer. N Engl J Med. 2013;368(11):987-98.

7. Shimizu Y, Kodama K, Nishi N, Kasagi F, Suyama A, Soda M, et al. Radiation exposure and circulatory disease risk: Hiroshima and Nagasaki atomic bomb survivor data, 1950-2003. BMJ. 2010;340:b5349.

8. McGale P, Darby SC, Hall P, Adolfsson J, Bengtsson NO, Bennet AM, et al. Incidence of heart disease in 35,000 women treated with radiotherapy for breast cancer in Denmark and Sweden. Radiother 
Oncol. 2011;100(2):167-75.

9. Taunk NK, Haffty BG, Kostis JB, Goyal S. Radiation-induced heart disease: pathologic abnormalities and putative mechanisms. Front Oncol. 2015;5:39.

10. Ping Z, Peng Y, Lang H, Xinyong C, Zhiyi Z, Xiaocheng W, et al. Oxidative Stress in Radiation-Induced Cardiotoxicity. Oxid Med Cell Longev. 2020;2020:3579143.

11. Livingston K, Schlaak RA, Puckett LL, Bergom C. The Role of Mitochondrial Dysfunction in RadiationInduced Heart Disease: From Bench to Bedside. Front Cardiovasc Med. 2020;7:20.

12. Bhattacharya S, Asaithamby A. Ionizing radiation and heart risks. Semin Cell Dev Biol. 2016;58:1425.

13. Oldershaw R, Owens WA, Sutherland R, Linney M, Liddle R, Magana L, et al. Human CardiacMesenchymal Stem Cell-Like Cells, a Novel Cell Population with Therapeutic Potential. Stem Cells Dev. 2019;28(9):593-607.

14. Rubin P, Casarett GW. Clinical radiation pathology as applied to curative radiotherapy. Cancer. 1968;22(4):767-78.

15. Phinney DG, Pittenger MF. Concise Review: MSC-Derived Exosomes for Cell-Free Therapy. Stem Cells. 2017;35(4):851-8.

16. Zhu LP, Tian T, Wang JY, He JN, Chen T, Pan M, et al. Hypoxia-elicited mesenchymal stem cell-derived exosomes facilitates cardiac repair through miR-125b-mediated prevention of cell death in myocardial infarction. Theranostics. 2018;8(22):6163-77.

17. Fuchi N, Miura K, Doi H, Li TS, Masuzaki H. Feasibility of placenta-derived mesenchymal stem cells as a tool for studying pregnancy-related disorders. Sci Rep. 2017;7:46220.

18. Luo L, Urata Y, Yan C, Hasan AS, Goto S, Guo CY, et al. Radiation Exposure Decreases the Quantity and Quality of Cardiac Stem Cells in Mice. PLoS One. 2016;11(5):e0152179.

19. Di Trapani M, Bassi G, Midolo M, Gatti A, Kamga PT, Cassaro A, et al. Differential and transferable modulatory effects of mesenchymal stromal cell-derived extracellular vesicles on T, B and NK cell functions. Sci Rep. 2016;6:24120.

20. McBride WH, Schaue D. Radiation-induced tissue damage and response. J Pathol. 2020;250(5):64755.

21. Manda K, Kavanagh JN, Buttler D, Prise KM, Hildebrandt G. Low dose effects of ionizing radiation on normal tissue stem cells. Mutat Res. 2014;761:6-14.

22. Ward MR, Abadeh A, Connelly KA. Concise Review: Rational Use of Mesenchymal Stem Cells in the Treatment of Ischemic Heart Disease. Stem Cells TransI Med. 2018;7(7):543-50.

23. Rossini A, Frati C, Lagrasta C, Graiani G, Scopece A, Cavalli S, et al. Human cardiac and bone marrow stromal cells exhibit distinctive properties related to their origin. Cardiovasc Res. 2011;89(3):650-60.

24. Sommariva E, Brambilla S, Carbucicchio C, Gambini E, Meraviglia V, Dello Russo A, et al. Cardiac mesenchymal stromal cells are a source of adipocytes in arrhythmogenic cardiomyopathy. Eur Heart J. 2016;37(23):1835-46. 
25. Marote A, Teixeira FG, Mendes-Pinheiro B, Salgado AJ. MSCs-Derived Exosomes: Cell-Secreted Nanovesicles with Regenerative Potential. Front Pharmacol. 2016;7:231.

26. Alcayaga-Miranda F, Varas-Godoy M, Khoury M. Harnessing the Angiogenic Potential of Stem CellDerived Exosomes for Vascular Regeneration. Stem Cells Int. 2016;2016:3409169.

27. Deo M, Weinberg SH, Boyle PM. Calcium Dynamics and Cardiac Arrhythmia. Clin Med Insights Cardiol. 2017;11:1179546817739523.

28. Meng Y, Li WZ, Shi YW, Zhou BF, Ma R, Li WP. Danshensu protects against ischemia/reperfusion injury and inhibits the apoptosis of H9c2 cells by reducing the calcium overload through the p-JNKNF-kappaB-TRPC6 pathway. Int J Mol Med. 2016;37(1):258-66.

29. Hu SY, Zhang Y, Zhu PJ, Zhou H, Chen YD. Liraglutide directly protects cardiomyocytes against reperfusion injury possibly via modulation of intracellular calcium homeostasis. J Geriatr Cardiol. 2017;14(1):57-66.

30. Geng Z, Xu F, Zhang Y. MiR-129-5p-mediated Beclin-1 suppression inhibits endothelial cell autophagy in atherosclerosis. Am J Transl Res. 2016;8(4):1886-94.

31. Zhao H, Song X, Yan L, Ren M, Cui X, Li Y, et al. IgE induces hypotension in asthma mice by downregulating vascular NCX1 expression through activating MiR-212-5p. Biochim Biophys Acta Mol Basis Dis. 2018;1864(1):189-96.

32. Li WD, Zhou DM, Sun LL, Xiao L, Liu Z, Zhou M, et al. LncRNA WTAPP1 Promotes Migration and Angiogenesis of Endothelial Progenitor Cells via MMP1 Through MicroRNA 3120 and Akt/PI3K/Autophagy Pathways. Stem Cells. 2018;36(12):1863-74.

33. Ge Y, Wang J, Wu D, Zhou Y, Qiu S, Chen J, et al. IncRNA NR_038323 Suppresses Renal Fibrosis in Diabetic Nephropathy by Targeting the miR-324-3p/DUSP1 Axis. Mol Ther Nucleic Acids. 2019;17:741-53.

34. Guo J, Sang Y, Yin T, Wang B, Yang W, Li X, et al. miR-1273g-3p participates in acute glucose fluctuation-induced autophagy, dysfunction, and proliferation attenuation in human umbilical vein endothelial cells. Am J Physiol Endocrinol Metab. 2016;310(9):E734-43.

35. Yang S, Ye ZM, Chen S, Luo XY, Chen SL, Mao L, et al. MicroRNA-23a-5p promotes atherosclerotic plaque progression and vulnerability by repressing ATP-binding cassette transporter A1/G1 in macrophages. J Mol Cell Cardiol. 2018;123:139-49.

36. Dong Z, Li S, Si L, Ma R, Bao L, Bo A. Identification IncRNA LOC102551149/miR-23a-5p pathway in hepatic fibrosis. Eur J Clin Invest. 2020;50(6):e13243.

37. Brea R, Motino O, Frances D, Garcia-Monzon C, Vargas J, Fernandez-Velasco M, et al. PGE2 induces apoptosis of hepatic stellate cells and attenuates liver fibrosis in mice by downregulating miR-23a-5p and miR-28a-5p. Biochim Biophys Acta Mol Basis Dis. 2018;1864(2):325-37.

38. Lu L, McCurdy S, Huang S, Zhu X, Peplowska K, Tiirikainen M, et al. Time Series miRNA-mRNA integrated analysis reveals critical miRNAs and targets in macrophage polarization. Sci Rep. 2016;6:37446. 
39. Zhang S, Yin Z, Dai FF, Wang H, Zhou MJ, Yang MH, et al. miR-29a attenuates cardiac hypertrophy through inhibition of PPARdelta expression. J Cell Physiol. 2019;234(8):13252-62.

40. Li M, Wang N, Zhang J, He HP, Gong HQ, Zhang R, et al. MicroRNA-29a-3p attenuates ET-1-induced hypertrophic responses in H9c2 cardiomyocytes. Gene. 2016;585(1):44-50.

41. Zhang L, Zhang J, Tong Q, Wang G, Dong H, Wang Z, et al. Reduction of miR-29a-3p induced cardiac ischemia reperfusion injury in mice via targeting Bax. Exp Ther Med. 2019;18(3):1729-37.

42. Cheng HS, Sivachandran N, Lau A, Boudreau E, Zhao JL, Baltimore D, et al. MicroRNA-146 represses endothelial activation by inhibiting pro-inflammatory pathways. EMBO Mol Med. 2013;5(7):1017-34.

43. Pan JA, Tang Y, Yu JY, Zhang H, Zhang JF, Wang CQ, et al. miR-146a attenuates apoptosis and modulates autophagy by targeting TAF9b/P53 pathway in doxorubicin-induced cardiotoxicity. Cell Death Dis. 2019;10(9):668.

44. Ibrahim AG, Cheng K, Marban E. Exosomes as critical agents of cardiac regeneration triggered by cell therapy. Stem Cell Reports. 2014;2(5):606-19.

45. Oh JG, Watanabe S, Lee A, Gorski PA, Lee P, Jeong D, et al. miR-146a Suppresses SUMO1 Expression and Induces Cardiac Dysfunction in Maladaptive Hypertrophy. Circ Res. 2018;123(6):673-85.

46. Fang L, Ellims AH, Moore XL, White DA, Taylor AJ, Chin-Dusting J, et al. Circulating microRNAs as biomarkers for diffuse myocardial fibrosis in patients with hypertrophic cardiomyopathy. J Transl Med. 2015;13:314.

47. Brooks AL, Hoel DG, Preston RJ. The role of dose rate in radiation cancer risk: evaluating the effect of dose rate at the molecular, cellular and tissue levels using key events in critical pathways following exposure to low LET radiation. Int J Radiat Biol. 2016;92(8):405-26.

48. Averbeck D, Salomaa S, Bouffler S, Ottolenghi A, Smyth V, Sabatier L. Progress in low dose health risk research: Novel effects and new concepts in low dose radiobiology. Mutat Res. 2018;776:46-69.

\section{Tables}

Table 1. The top 20 miRNAs that up-regulated in exosomes from irradiated hp-MSCs (versus nonirradiated hp-MSCs). 


\begin{tabular}{|llll|}
\hline miRNA & Ratio: Irradiated-exo to & Irradiated-exo & Non-irradiated-exo \\
& Non-irradiated-exo & & \\
\hline hsa-miR-4655-5p & 3.20 & 15.08 & 4.71 \\
\hline hsa-miR-6506-5p & 2.92 & 9.08 & 3.11 \\
\hline hsa-miR-4635 & 2.89 & 13.53 & 4.68 \\
\hline hsa-miR-129-5p & 2.81 & 7.13 & 2.54 \\
\hline hsa-miR-6772-3p & 2.81 & 6.10 & 2.17 \\
\hline hsa-mir-3689b & 2.81 & 10.45 & 3.72 \\
\hline hsa-miR-212-5p & 2.71 & 8.65 & 3.20 \\
\hline hsa-miR-3157-3p & 2.60 & 8.48 & 3.26 \\
\hline hsa-miR-3120-5p & 2.35 & 6.55 & 2.78 \\
\hline hsa-miR-16-1-3p & 2.35 & 7.59 & 3.24 \\
\hline hsa-miR-4638-5p & 2.32 & 7.65 & 3.29 \\
\hline hsa-miR-2392 & 2.32 & 6.18 & 2.67 \\
\hline hsa-miR-4330 & 2.31 & 4.98 & 2.16 \\
\hline hsa-miR-3652 & 2.26 & 7.15 & 3.17 \\
\hline hsa-miR-4686 & 2.22 & 6.34 & 2.86 \\
\hline hsa-miR-7850-5p & 2.22 & 5.42 & 2.44 \\
\hline hsa-miR-324-3p & 2.15 & 20.76 & 9.65 \\
\hline hsa-miR-4535 & 2.15 & 12.89 & 6.01 \\
\hline hsa-miR-4538 & 2.13 & 13.57 & 6.36 \\
\hline hsa-miR-5087 & 2.12 & 9.64 & 4.55 \\
\hline
\end{tabular}

Non-irradiated-exo: exosomes isolated from conditioned medium of non-irradiated hp-MSCs, Irradiatedexo: exosomes isolated from conditioned medium of irradiated hp-MSCs.

Table 2. The top 20 miRNAs that down-regulated in exosomes from irradiated hp-MSCs (versus nonirradiated hp-MSCs). 


\begin{tabular}{|c|c|c|c|}
\hline miRNA & $\begin{array}{l}\text { Ratio: Irradiated-exo to Non-irradiated- } \\
\text { exo }\end{array}$ & Irradiated-exo & Non-irradiated-exo \\
\hline hsa-miR-4443 & 0.25 & 10.98 & 44.66 \\
\hline hsa-miR-7110-5p & 0.26 & 55.08 & 212.58 \\
\hline hsa-let-7a-5p & 0.28 & 2.15 & 7.66 \\
\hline hsa-miR-520g-3p & 0.29 & 6.96 & 23.87 \\
\hline hsa-miR-382-5p & 0.30 & 5.37 & 18.20 \\
\hline hsa-miR-424-3p & 0.30 & 8.59 & 28.78 \\
\hline hsa-miR-3197 & 0.31 & 6.71 & 21.56 \\
\hline hsa-miR-6824-5p & 0.33 & 4.81 & 14.62 \\
\hline hsa-miR-3178 & 0.34 & 204.21 & 600.47 \\
\hline hsa-miR-32-3p & 0.35 & 3.96 & 11.39 \\
\hline $\begin{array}{l}\text { hsa-miR-1273g- } \\
3 p\end{array}$ & 0.35 & 352.65 & 1008.29 \\
\hline hsa-miR-23a-5p & 0.35 & 4.19 & 11.93 \\
\hline hsa-miR-29a-3p & 0.36 & 8.09 & 22.58 \\
\hline hsa-miR-3663-5p & 0.36 & 7.25 & 20.14 \\
\hline hsa-miR-146a-5p & 0.36 & 1.65 & 4.56 \\
\hline hsa-miR-517a-3p & 0.38 & 3.51 & 9.29 \\
\hline hsa-miR-517b-3p & 0.38 & 3.51 & 9.29 \\
\hline hsa-miR-3190-5p & 0.38 & 3.25 & 8.50 \\
\hline hsa-miR-516b-5p & 0.38 & 6.76 & 17.66 \\
\hline hsa-miR-4692 & 0.38 & 2.08 & 5.42 \\
\hline
\end{tabular}

Non-irradiated-exo: exosomes isolated from conditioned medium of non-irradiated hp-MSCs, Irradiatedexo: exosomes isolated from conditioned medium of irradiated hp-MSCs.

\section{Figures}


A

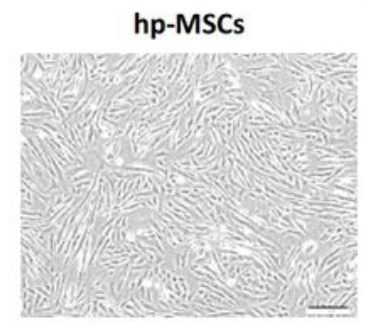

C

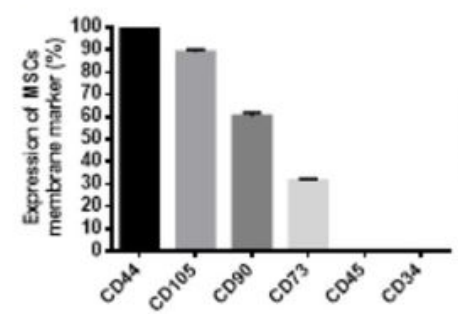

B
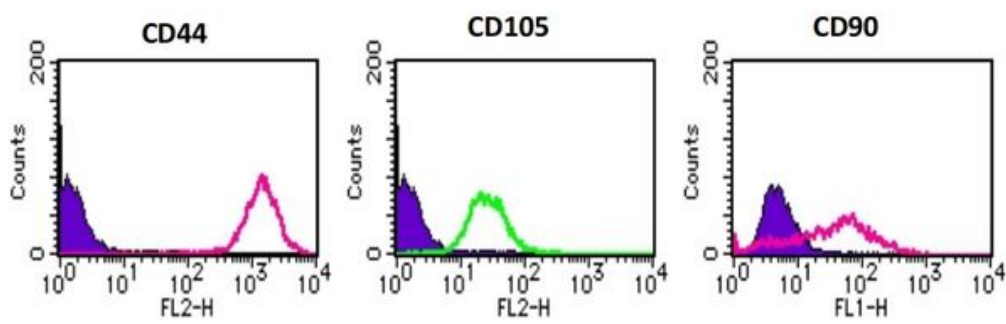

CD45
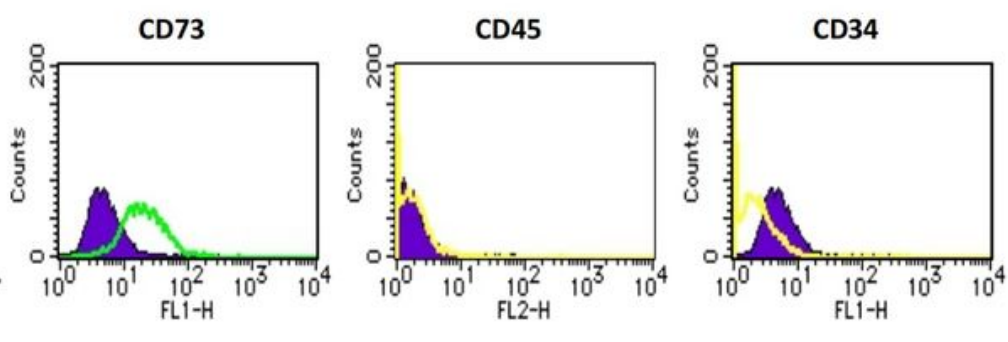

D

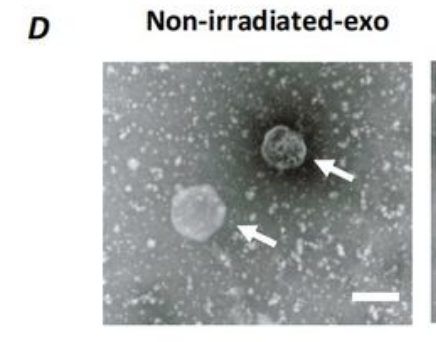

F
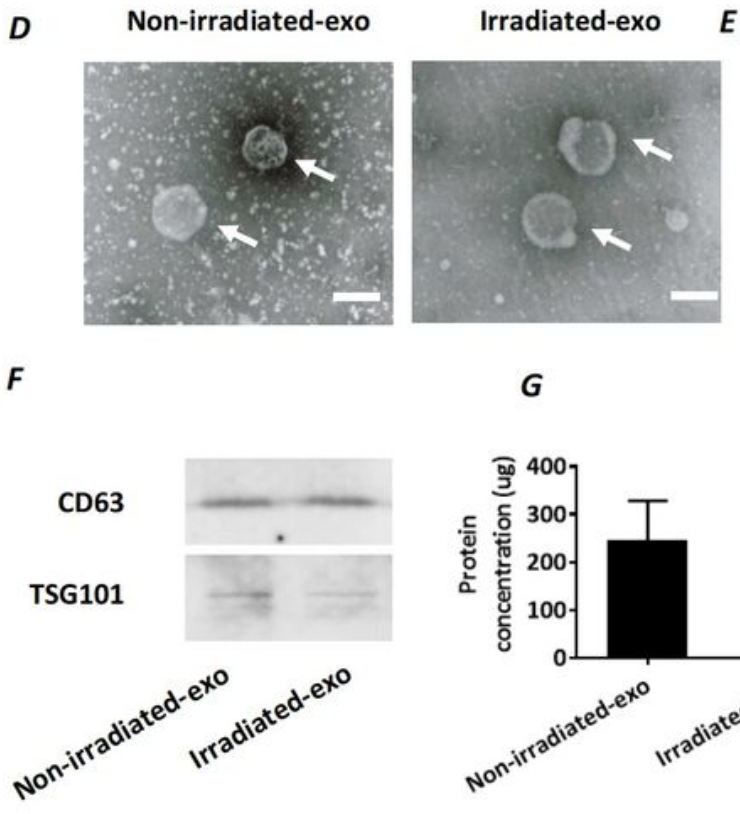

G

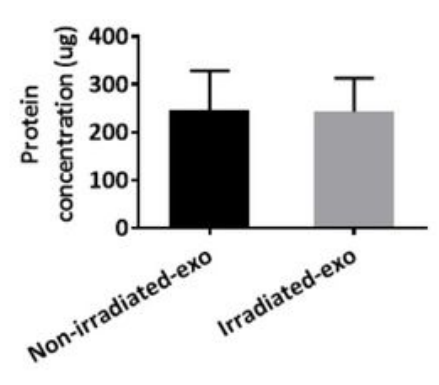

Irradiated-exo

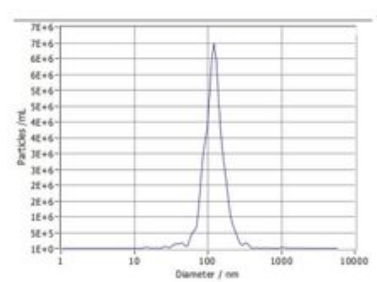

H

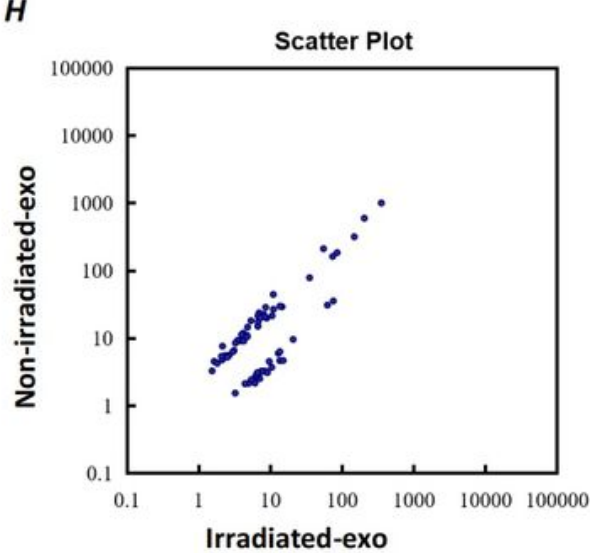

\section{Figure 1}

Characterization of hp-MSCs and hp-MSCs exosomes. (A) Human placenta tissues-derived mesenchymal stem cells (hp-MSCs) displayed identical fibroblast morphology. Representative images are shown. Scale bar: $200 \mu \mathrm{m}$. Representative histograms (B) and quantitative data (C) of flow cytometry analysis about the expressions of CD44, CD105, CD90, and CD73, but not CD45 and CD34 in hp-MSCs. (D) Representative images from electron microscope show the exosomes (white arrow) from non-irradiated 
and irradiated hp-MSCs. Scale bar: $100 \mathrm{~nm}$. (E) Nanoparticle track analysis on the size-distribution of exosomes from non-irradiated and irradiated hp-MSCs. (F) Western blotting analysis of CD63 and TSG101 expressions of exosomes from non-irradiated and irradiated hp-MSCs. (G) Protein concentration of exosomes from non-irradiated and irradiated hp-MSCs determined by BCA protein assay. $(\mathrm{H}) \mathrm{Scatter}$ plot of miRNA expressions in exosomes from non-irradiated versus irradiated hp-MSCs. Values are the mean $\pm S D(n=3)$. Non-irradiated-exo: exosomes isolated from conditioned medium of non-irradiated hpMSCs, Irradiated-exo: exosomes isolated from conditioned medium of irradiated hp-MSCs.

$A$

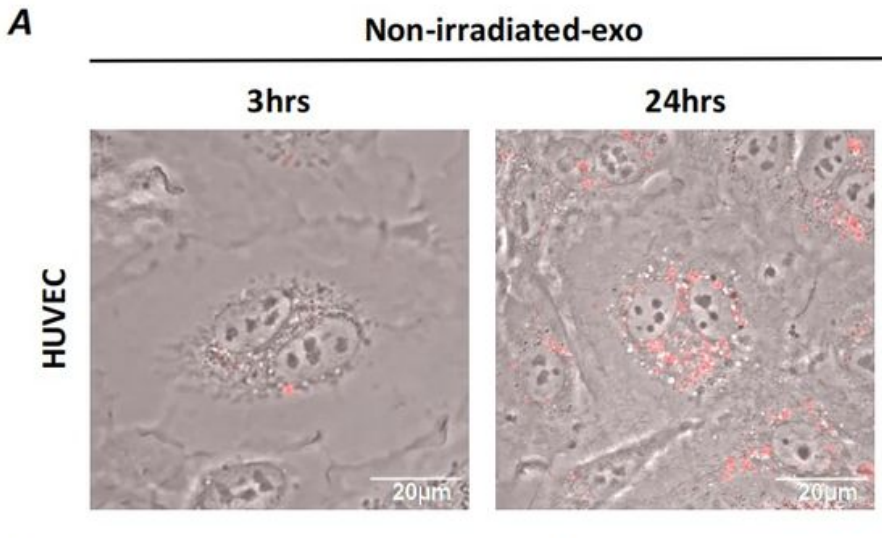

Non-irradiated-exo

Irradiated-exo

B

Non-irradiated-exo
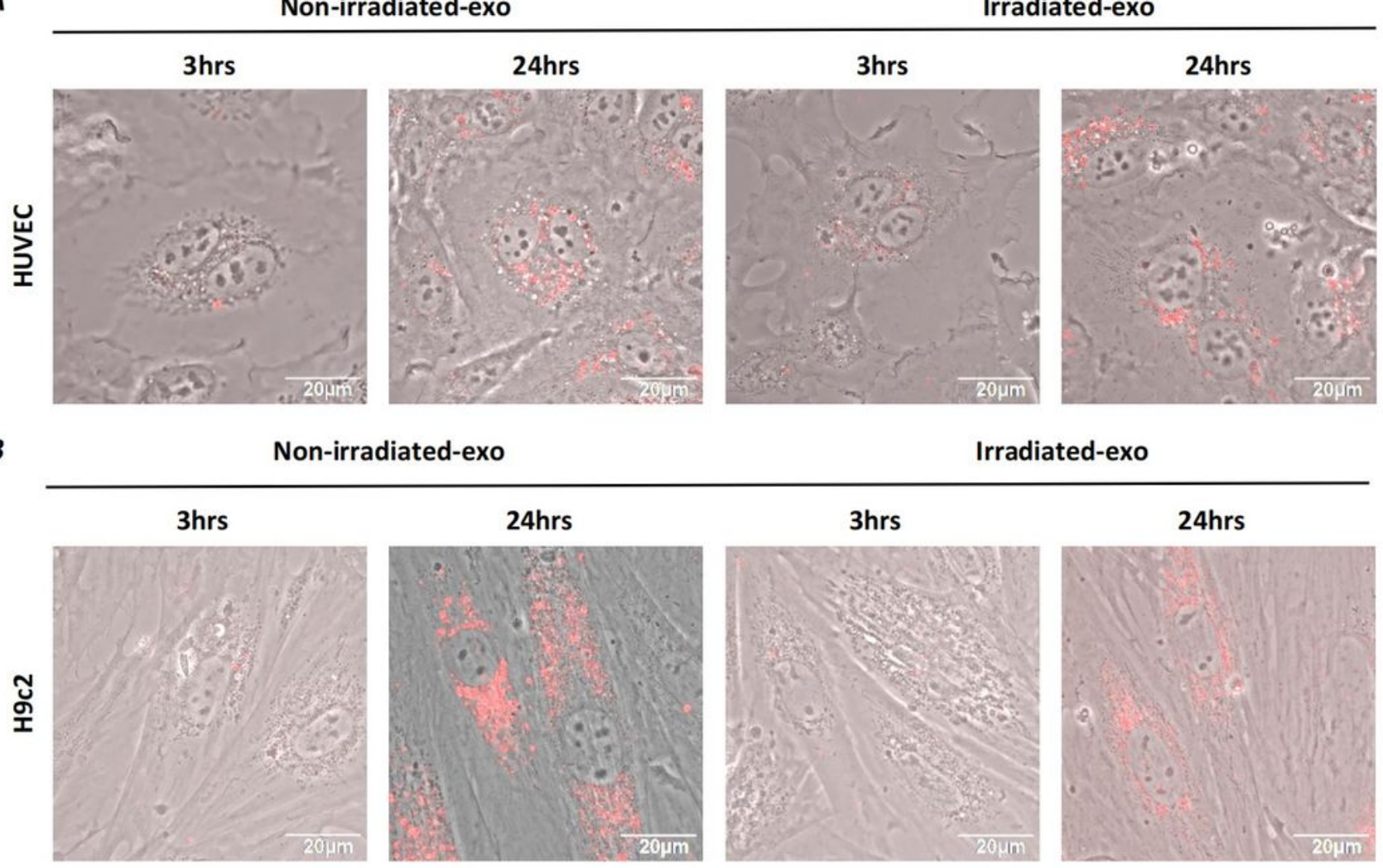

24hrs

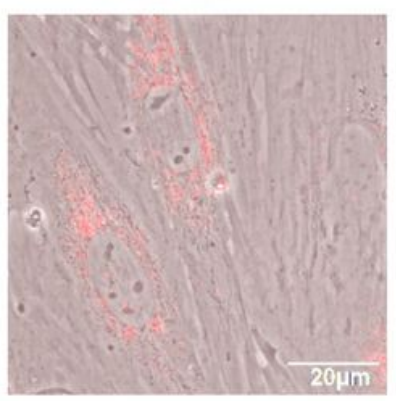

\section{Figure 2}

The uptake of hp-MSCs derived exosomes by HUVEC and H9c2 cells. Confocal images of HUVEC (A) and H9c2 cells (B) with internalized PKH26 labeled exosomes from non-irradiated/irradiated hp-MSCs after 3 or 24 hours of co-culture. Scale bar: $20 \mu \mathrm{m}$. 

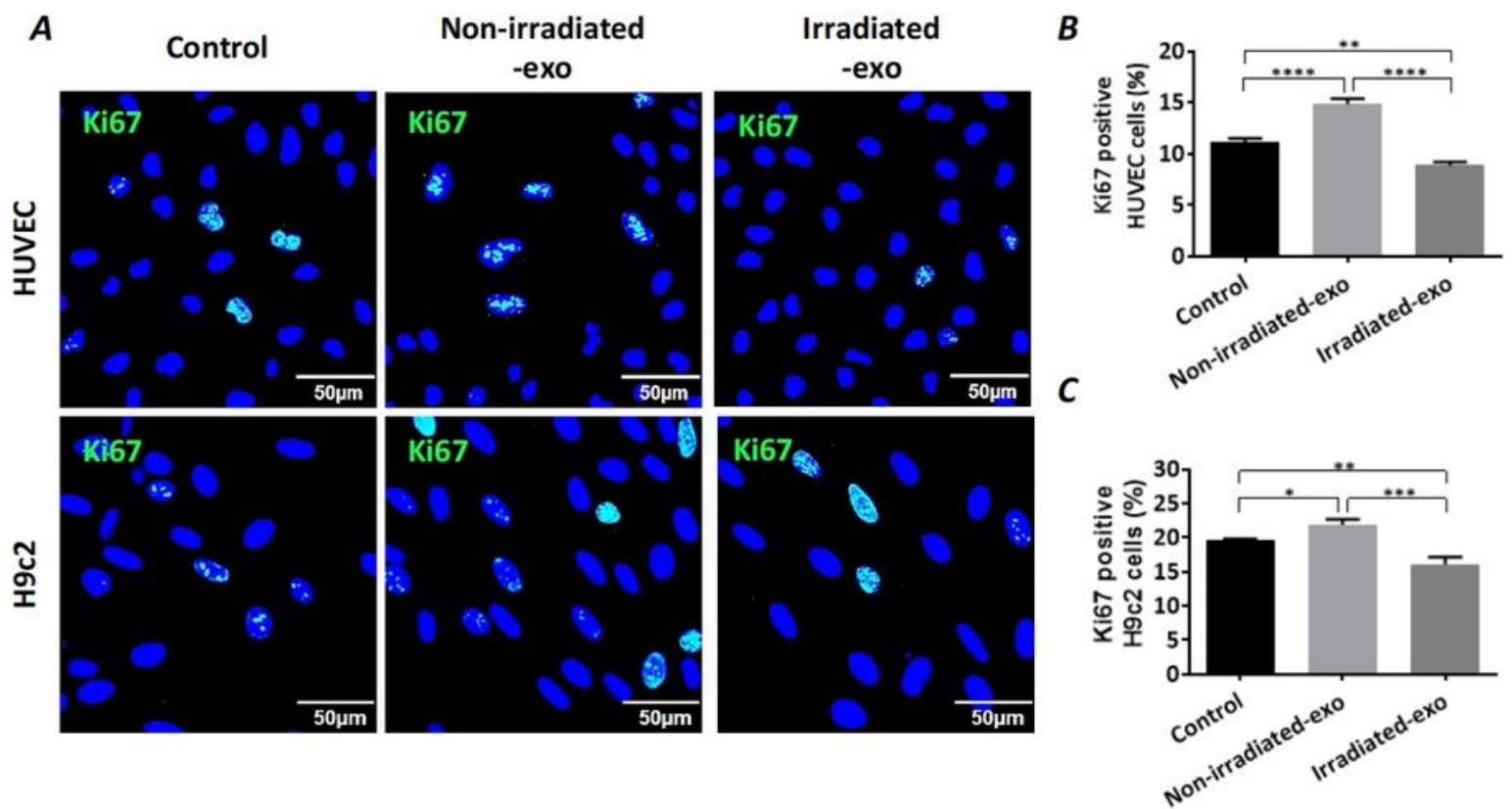

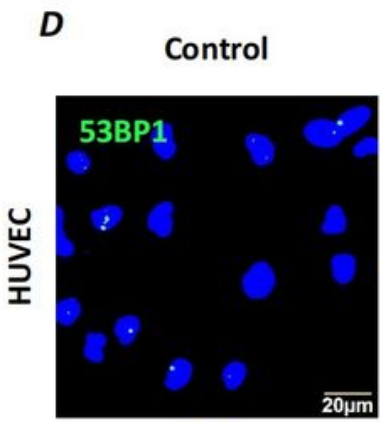

Non-irradiated

-exo
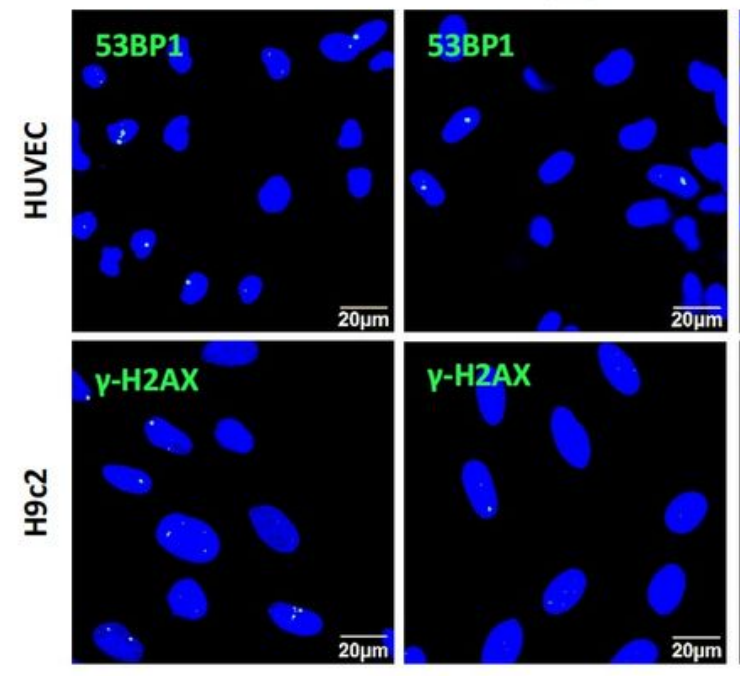

\section{Irradiated}

-exo

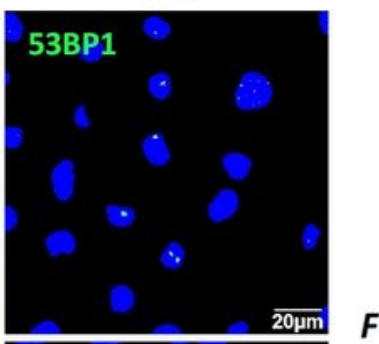

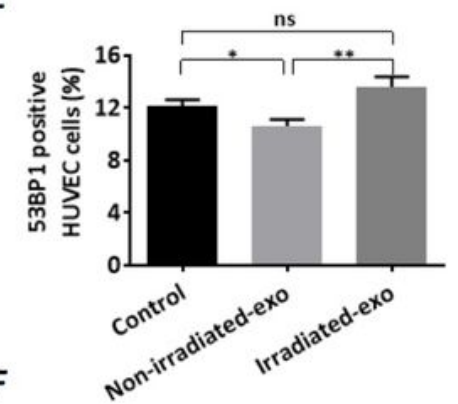

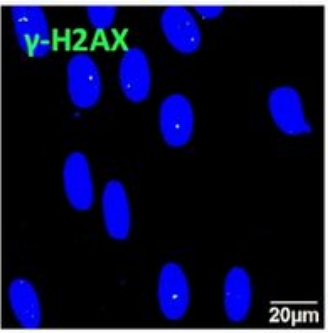

\section{Figure 3}

Effects of hp-MSCs derived exosomes on the proliferation and DNA damage of HUVEC and H9c2 cells. (A) Representative images of immunofluorescence analysis show the Ki67-positive cells in HUVEC or H9c2 cells. Scale bar: $50 \mu \mathrm{m}$. Quantitative data indicate the percentage of Ki67-positive cells in HUVEC cells (B) or H9c2 cells (C). (D) Representative images of immunofluorescence analysis show the DNA damage in HUVEC (53BP1 foci) or H9c2 (y-H2AX foci) cells. Scale bar: $20 \mu \mathrm{m}$. Quantitative data on the 
percentage of 53BP1-positive HUVEC cells (E) and $\mathrm{Y}$-H2AX-positive H9c2 cells (F). Non-irradiated-exo: exosomes isolated from conditioned medium of non-irradiated hp-MSCs, Irradiated-exo: exosomes isolated from conditioned medium of irradiated hp-MSCs. Values are the mean $\pm S D(n=3)$. ns $P>0.5$, *P

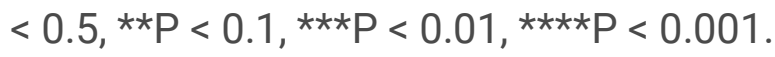

$A$
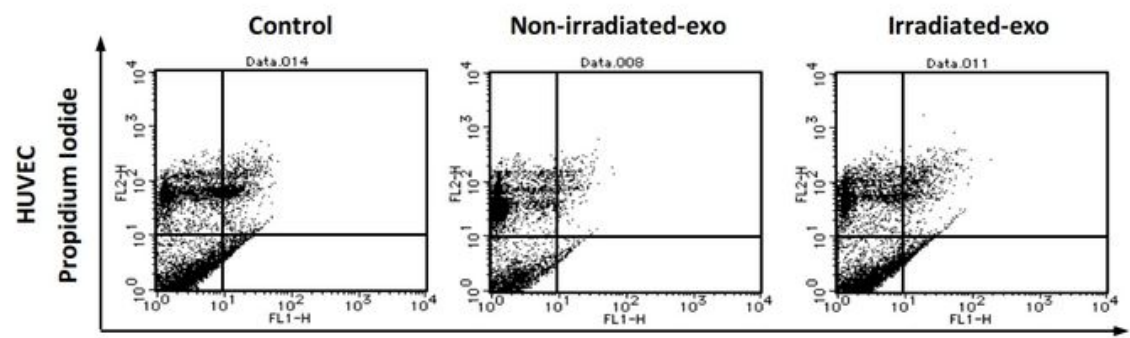

Annexin V

B

$C$
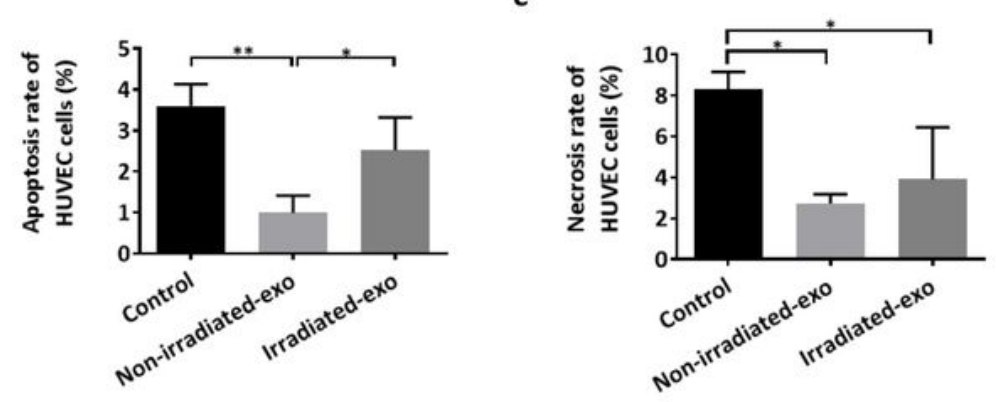

D
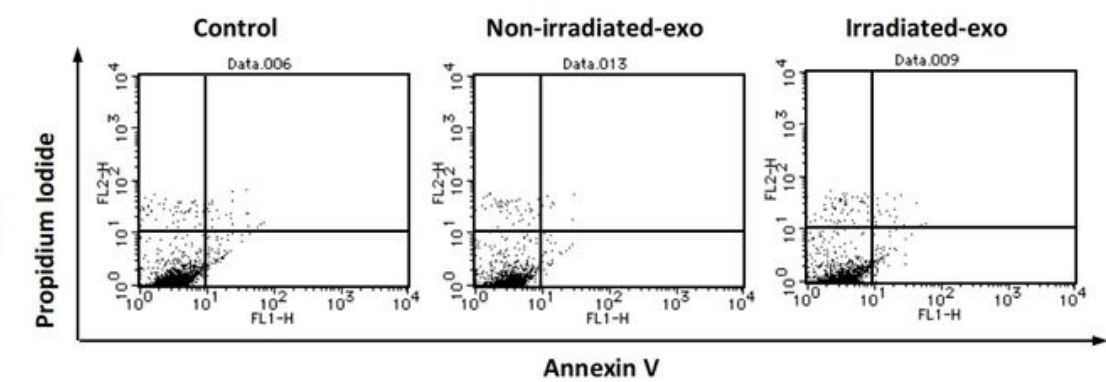

$E$

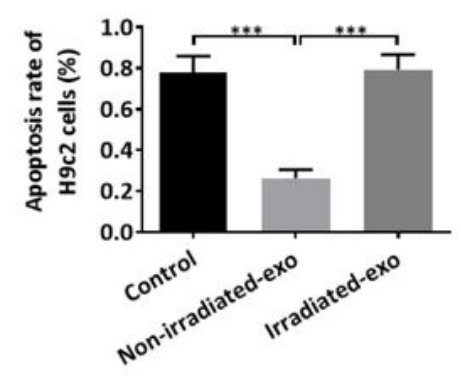

F

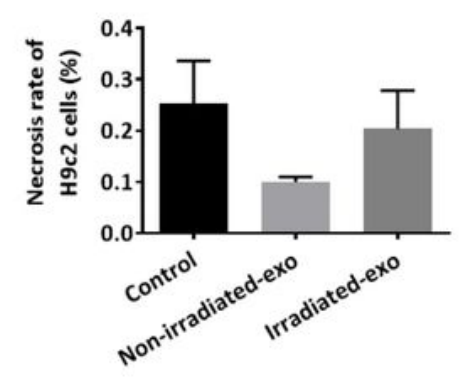

Figure 4 
Effects of hp-MSCs derived exosomes on the apoptosis of HUVEC and H9c2 cells. (A) Representative dot plots of flow cytometry analysis on the apoptosis of HUVEC cells. Quantitative data on the annexin-Vpositive apoptotic HUVEC cells (B) and propidium iodide-labeled necrotic HUVEC cells (C). (D) Representative dot plots of flow cytometry analysis on the apoptosis of H9c2 cells. Quantitative data on the apoptosis $(\mathrm{E})$ and necrosis $(\mathrm{F})$ of $\mathrm{H} 9 \mathrm{c} 2$ cells. Non-irradiated-exo: exosomes isolated from conditioned medium of non-irradiated hp-MSCs, Irradiated-exo: exosomes isolated from conditioned medium of irradiated hp-MSCs. Values are the mean $\pm S D(n=3) . * P<0.5, * * P<0.1, * \star * P<0.01$.

$\boldsymbol{A}$
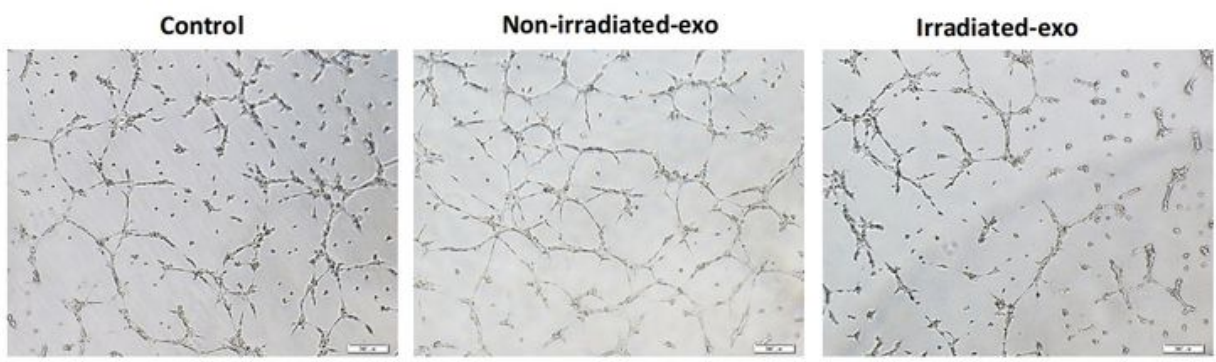

B

$c$
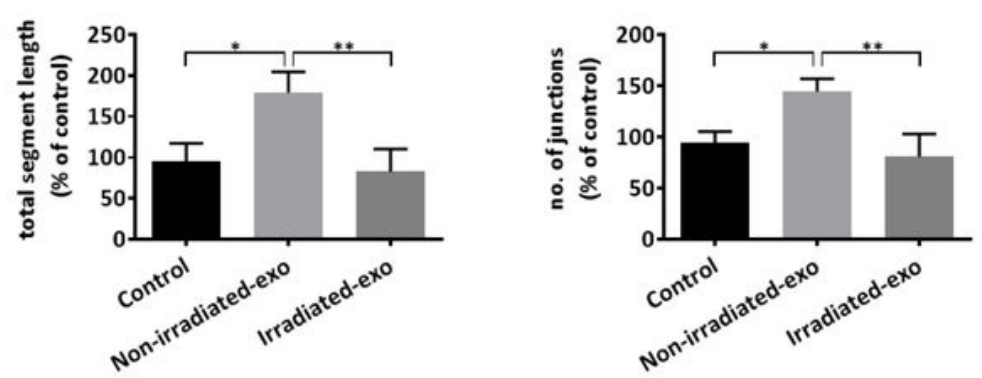

D

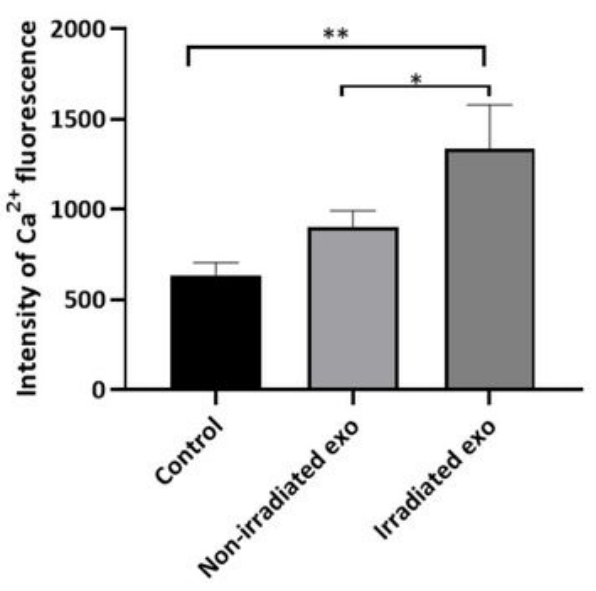




\section{Figure 5}

Effects of hp-MSCs derived exosomes on tube formation of HUVEC cells and calcium transient of H9c2 cells. (A) Representative images of vessel-like structures formation on Matrigel. HUVEC cells was previously co-cultured with exosomes or not for $48 \mathrm{~h}$. Scale bar: $200 \mu \mathrm{m}$. (B, C) Quantitative data on the tube formation relative to non-treated cells. (D) Quantitative analyses on the calcium fluorescence intensity in H9c2 cells co-cultured with exosomes from non-irradiated/irradiated hp-MSCs or not for $48 \mathrm{~h}$. Non-irradiated-exo: exosomes isolated from conditioned medium of non-irradiated hp-MSCs, Irradiatedexo: exosomes isolated from conditioned medium of irradiated hp-MSCs. Values are the mean \pm SD $(n=3) . n s P>0.5, * P<0.5, * * P<0.1$. 